\title{
Tangence
}

\section{Entre le récit de la fondation et le récit de l'autre : l'intertextualité dans Don Quichotte de la démanche}

\section{André Lamontagne}

Numéro 41, octobre 1993

Interdiscurtivité dans l’œuvre de Victor-Lévy Beaulieu

URI : https://id.erudit.org/iderudit/025775ar

DOI : https://doi.org/10.7202/025775ar

Aller au sommaire du numéro

Éditeur(s)

Tangence

ISSN

0226-9554 (imprimé)

1710-0305 (numérique)

Découvrir la revue

Citer cet article

Lamontagne, A. (1993). Entre le récit de la fondation et le récit de l'autre :

l'intertextualité dans Don Quichotte de la démanche. Tangence, (41), 32-42.

https://doi.org/10.7202/025775ar d'utilisation que vous pouvez consulter en ligne. 


\section{Entre le récit de fondation et le récit de l'autre: I'intertextualité dans Don Quichotte de la démanche}

\section{André Lamontagne *}

Un cochon qui avale un exemplaire de l'Ulysse de Joyce; "Balzac sur le dos, Hugo entrouvert et grotesque devant la chambre de bains" ${ }^{1}$; Don Quichotte qui fait une entrée intempestive dans le bungalow d'Abel Beauchemin pour lui tenir des propos lumineux sur le destin de la littérature québécoise; Ferron, Miron et Ducharme qui font procession rue SainteCatherine: une interprétation rapide s'autoriserait à lire les facéties intertextuelles présentes dans Don Quichotte de la démanche en fonction de la poétique postmoderne. Mais ce serait négliger deux détails: d'une part, l'absence d'une tradition postmoderne québécoise en 1974, date de parution de ce roman de Victor-Lévy Beaulieu, et, d'autre part, l'inscription dans ce même texte d'un récit et d'un projet politiques, traits modernistes difficilement compatibles avec les thèses d'un Lyotard, qui postulent la mort de l'État-nation et la remise en cause de l'Histoire.

L'on pourrait par ailleurs soutenir une lecture inverse, voir dans ce roman un projet moderniste "avorté "où le désir d'un récit national serait grevé par les mots des autres. Don Quichotte de la démanche reconduirait ainsi le conflit des codes tel que défini par André Belleau, où l'intertexte français est appelé à signaler la légitimité littéraire du roman québécois, mais sans pour autant s'intégrer à une diégèse caractérisée par le code socio-culturel de l'authentique, porteur d'une méfiance à l'égard de la culture $^{2}$. Mais on passerait alors sous silence la diversification de l'intertextualité, qui n'est plus essentiellement française,

André Lamontagne est professeur à l'Université de ColombieBritannique.

1 Victor-Lévy Beaulieu, Don Quichotte de la démanche, Montréal, Éditions de L'Aurore, 1974, p. 65.

2 Cf. "Le conflit des codes dans l'institution littéraire québécoise", Surprendre les voix, Boréal, 1986, p. 167-174; "Code social et code littéraire dans le roman québécois", op. cit., p. 175-192. 
ainsi que la modification de sa fonction, qui n'est plus essentiellement discursive.

La saisie de l'intertextualité s'avère donc problématique dans Don Quichotte de la Démanche, et j'y vois le signe que quelque chose a changé dans la littérature québécoise et que quelque chose de fondamental persiste. L'hypothèse que je pose, et que j'ai déjà entrepris de vérifier dans des œuvres telles Prochain épisode d'Hubert Aquin et Maryse de Francine Noël, est celle-ci: l'évolution de la dynamique intertextuelle du roman québécois consacre son passage du modernisme au postmodernisme, mais tout en avivant une problématique identitaire qui fonde une spécificité, qui résiste à la visée globalisante du discours critique sur la fiction contemporaine.

Résumé très grossièrement, Don Quichotte de la démanche raconte deux nuits et une journée au cours desquelles l'écrivain Abel Beauchemin est envahi de toutes parts par son imaginaire, livré aux démons de son œuvre, de son passé, du gros gin et à la nostalgie de sa femme Judith, qui l'a quitté. Entre ces deux nuits marquées par une crise d'angoisse et une visite à l'hôpital s'insèrent quelques événements diurnes. Mais l'essentiel du roman consiste en des séances d'écriture et en une vaste réflexion sur la vie, la mort et le sens de la création artistique.

Du projet littéraire ponctuel que poursuit Abel Beauchemin, on sait somme toute peu de choses ou alors trop: tel Orphée, il écrit pour faire revenir Judith; il cherche également à faire "le récit du bébé" qu'elle n'aura pas avec lui; il mène une séance d'“écriture-exorcisme" pour expier une faute d'enfance; il amorce les romans de Jos et de Géronimo; et il voudrait écrire sur Steven et son père mais en est incapable. Ce qui se profile derrière ces projets en apparence disjoints, ce qui survit au sentiment d'impuissance littéraire qui gagne Abel toutes les nuits, c'est bel et bien le grand CEuvre épique «qui ferait s'ébranler enfin les pays québécois" (p. 239). Mais, pour reprendre la question posée par Jacques Pelletier: "comment, en somme, tirer l'Euvre de la nonhistoire, du magma informe, de la béance (an)historique?." 3 Abel ou VLB pourrait répondre: en écrivant l'histoire de la Grande

3 Jacques Pelletier, "Victor-Lévy Beaulieu. De la vraie saga des Beauchemin aux querelles d'héritage des Galarneau: la quête de l'épopée", Le roman national, VLB éditeur, coll. "Essais critiques", 1991, p. 180. 
34

Tribu, en proposant un récit familial qui est un avatar du récit de fondation. Comme l'écrit Jacques Michon:

À défaut de pays ou d'État qui pourrait donner lieu à des sujets responsables libérés des anciens atavismes, des devoirs familiaux, des vendetta à répétition, l'histoire s'écrirait donc comme un roman familial. À pays équivoque famille certaine. ${ }^{4}$

Mais "l'extrême indigence dans laquelle il faut bien se débattre" (p. 158) n'est pas qu'historique, elle est également littéraire. Le récit de l'autre, l'intertextualité, vient donc se juxtaposer au récit familial pour combler un manque. À cet égard, un des cauchemars faits par Abel est très significatif. Dans ce rêve, les membres de la famille explorent la mémoire et l'inconscient d'Abel: ils découvrent les maisons d'enfance, la maison des ancêtres et aussi un édifice nommé "la Bibliothèque de Bibi" (p. 220). L'allégorie est limpide: la mémoire de l'écrivain est autant intertextuelle qu'historique ou familiale. Certes, après Borges, après Calvino, cela n'est pas nouveau. Mais dans le contexte québécois, cette dynamique prend un sens particulier, témoigne d'un changement dont les modalités me semblent rassemblées dans l'intervention de Don Quichotte à la fin du roman.

Il faut d'abord noter que cette apparition, qu'elle soit de l'ordre de l'hallucination ou du fantastique, est un acte diégétique: une figure de la lointaine culture peut désormais entrer dans l'univers d'un personnage romanesque québécois. Si l'on considère, en suivant André Belleau, que "la fonction de la référence littéraire, dans la plupart de nos romans, demeure essentiellement discursive, énonciative, aussi bien chez Jacques Godbout, romancier du discours social, que chez Réjean Ducharme, romancier du discours littéraire, ou Yolande Villemaire" 5 , le roman de VLB marque donc une rupture. On remarquera ensuite

4 Et le critique d'ajouter: "La famille serait la première organisation dans une société sans État, ou dans une société où la notion d'Etat n'aurait pas atteint une légitimité suffisante pour faire l'histoire, donner un sens ou un destin aux individus et permettre l'émergence, par exemple, sur le plan littéraire de l'épopée ou du grand roman bourgeois (Les misérables, Moby Dick) qui fait l'envie et le désespoir d'Abel. : Jacques Michon, "Projet littéraire et réalité romanesque d'Abel Beauchemin", Études françaises, vol. XIX, $\mathrm{n}^{\circ} 1$, printemps 1983, p. 21.

5 "Code social et code littéraire dans le roman québécois", Surprendre les voix, op. cit., p. 187. 
que le conflit des codes se voit également modifié en ce que les intertextes, par lesquels le roman québécois signalait son appartenance à la littérature savante, ne sont plus majoritairement tributaires du discours littéraire français: le "ciel du Québec" est plutôt peuplé par Cervantès, Joyce, Ferron, Miron, Ducharme et Archibald Scott. Au cosmopolitisme littéraire correspond donc un mouvement de légitimation et de consécration des écrivains québécois.

Cette configuration intertextuelle particulière est cependant marquée par des traits typiquement postmodernes. Comme il se doit, Don Quichotte est maladroit, quelque peu ridicule, mais en tant que figure de l'Écrivain, il participe d'une désacralisation du discours hégémonique qu'est la littérature. La parodie, le travestissement et l'ironie entrent également en jeu: la Manche devient le pays de la démanche; Rossinante est remplacée par Goulatromba, un cheval qui appartient à l'œuvre de Victor Hugo. Cette dynamique postmoderne est toutefois contrebalancée par la recherche d'un modèle d'autorité: "cela m'enthousiasme de penser qu'un pareil à moi-même pourrait venir au monde chez vous [...]. J'aimerais que vous ayez un héros qui serait pour vous ce que moi je fus du temps de mon Espagne. Tous les signes sont là" (p. 258). Corrélativement, on assiste à une valorisation du Texte national, mais ce récit de fondation s'écrirait aussi avec les textes des autres, de la culture universelle. Entre les mots d'ailleurs et ceux d'ici, il y a moins antagonisme que complicité, écho, complémentarité.

L'espace nous manque pour analyser de façon détaillée comment cette diversification de l'intertextualité, la modification de sa fonction ainsi que la remise en question des discours dominants informent le roman dans son ensemble. Il est quand même possible d'indiquer quelques pistes. Un relevé sommaire des textes et écrivains convoqués dans Don Quichotte permet effectivement de croire à la diversification. Outre Cervantès, Miron, Ferron, Ducharme, Balzac, Hugo et Joyce, on note la présence d'Homère, Virgile, Malcolm Lowry, Melville, Herman Broch, Faulkner, Burroughs, Zola, Flaubert, Freud, saint Thomas d'Aquin, Henry James, Aimé Césaire, Dostoïevski, Nelligan, Mallarmé, Shakespeare, ainsi que de la Bible et j'en passe. Bien que la plupart soient appelés par analogie sémantique, ces intertextes n'obéissent pas tous aux mêmes modalités. Certains demeurent au niveau du discours, comme lorsque le narrateur décrit Abel 
"sombrant comme le Pequod melvilien [sic] défoncé à coups de queue par la baleine blanche!" (p. 247) Certains sont narrativement motivés, comme les réminiscences littéraires, mais ont essentiellement une fonction de renvoi (filiation littéraire, effets de culture, etc.). Par contre, on retrouve des intertextes qui remplissent une véritable fonction diégétique, qui exercent des déterminations sur la conduite des personnages ou sur l'histoire.

Certains de ces actes diégétiques sont très concrets et ils participent de la remise en question de ce que Lyotard appelle les "métarécits de légitimation " 6 , et, au premier chef, la littérature. Ainsi, lors d'un de ses séjours à l'hôpital, Abel fait tomber par terre Germinal et Lumière d'août. L'infirmière ramasse les livres avec du papier hygiénique et constate: "Faut les stériliser. En attendant, je peux vous prêter mon recueil de mots croisés" (p. 14). André Belleau aurait sans doute vu dans cette phrase une illustration du sort qui est fait à la culture savante dans notre société. Mais cet épisode s'inscrit dans un mouvement plus global où les écrivains, et surtout ceux qui composent la bibliothèque d'Abel, sont rabaissés. Victor Hugo, qui figure très haut dans le panthéon littéraire de VLB, subit indirectement plusieurs affronts à travers le cheval Goulatromba, qui, sous forme de poster, se fait successivement ouvrir le ventre par les chats et brûler par Abel ${ }^{7}$. De la même façon, les chats maculent de boue les affiches de Guevara, Hemingway et Abraham Lincoln, alors qu'Abel renverse Balzac et jette par terre les Euvres completes de Flaubert. Ces deux gestes complémentaires - chosification des auteurs et personnification des livres - traduisent donc à la fois une démythification de l'écriture, qui à plusieurs reprises dans le roman est opposée à la vie, et une désacralisation des œuvres qui forment le canon de la littérature occidentale.

C'est dans le même esprit de banalisation, je crois, qu'il faut tout d'abord comprendre l'esthétique du plagiat qui traverse Don Quichotte de la démanche:

[Abel] pillait comme un barbare avec une bonne conscience quand il lui apparaissait urgent ou nécessaire de faire un lien,

6 Cf. Jean-François Lyotard, La condition postmoderne, Paris, Minuit, 1979; Le postmodernisme expliqué aux enfants, Paris, Galilée, 1986.

7 Dans La nuitte de Malcomm Hudd, rappelons-le, Goutalombra se fait assassiner, mais non plus figurativement cette fois, par Annabelle, la femme jalouse de Malcomm. 
d'amorcer une nouvelle situation. Par expérience, il savait que n'importe quelle phrase faisait alors l'affaire et qu'au lieu de perdre son temps à en imaginer une, il valait mieux la piquer dans l'un des ouvrages qu'il avait pris au hasard dans sa bibliothèque. Il trouvait le procédé excellent et expéditif, tout en étant fort reposant pour l'esprit. (p. 81)

Dans une entrevue accordée à Jacques Pelletier, Victor-Lévy Beaulieu réaffirmait cette fonction utilitaire du plagiat dans Don Quichotte de la démanche ${ }^{8}$. Sans doute faut-il le croire, mais il me semble pourtant que les emprunts, dans le roman, sont très souvent signalés. Un lecteur plus érudit, plus attentif ou moins pressé que moi arriverait peut-être à un résultat différent, mais la plupart des intertextes que j'ai pu identifier révèlent leur caractère citationnel: soit que le fragment provoque, pour reprendre le terme de Michael Riffaterre, une agrammaticalité sémantique ou stylistique qui attire notre attention, ou soit que le fragment appartienne à un texte très connu - par exemple, lorsque Jos demande à son employeur: "Hélie, Hélie, pourquoi mas-tu embauché" (p. 47). En fait, l'aveu de plagiat révèle une contradiction dans les termes et c'est pourquoi, en dernière instance, le plagiat désigne beaucoup plus le travail intertextuel que la contrefaçon. Comme l'indique VLB :

C'est dans ce sens-là [l'inspiration] que j'utilise ce mot-là et je ne vois pas pourquoi on aurait plus de respect qu'il faut pour la littérature qui a déjà existé, par exemple pourquoi ne pourrais-je pas m'approprier le Don Quichotte de Cervantès?"?

Ainsi, à la désacralisation se conjugue l'idée postmoderne de la littérature vue comme répétition, dont le prototype est la nouvelle de Borges intitulée "Pierre Ménard, auteur du Quichotte".

8 Jacques Pelletier, "Victor-Lévy Beaulieu écrivain professionnel", Voix et images, vol. III, $\mathrm{n}^{\circ} 2$, décembre 1977, p. 183. Dans cette entrevue, l'idée de l'écrivain comme être foncièrement amoral est attribuée à Faulkner, tandis que dans Don Quichotte de la démancbe Abraham Sturgeon prête cette phrase à William Burroughs. S'agit-là d'une erreur ou d'une fausse attribution à la Aquin?

9 Ibid. VLB ajoute: "Les critiques ont dit que je m'étais inspiré d'Audessous du volcan de Lowry pour le Don Quichotte. Moi, ça m'a fait bien rire, car la grande inspiration de ce roman-là, c'est le livre de Virgile. "Il n'en demeure pas moins que le personnage d'Abel emprunte beaucoup au héros de Lowry: écrivain obsessionnel, alcoolique, qui n'arrive plus à départager la réalité de l'imaginaire. 
38

L'intertextualité se combine également à l'intersexualité pour pervertir certains textes qui façonnent l'idéologie dominante. Ainsi, Abel propose une nouvelle version de "La belle au bois dormant " dans laquelle le héros sodomise la princesse endormie. Ailleurs, se remémorant une scène où il avait été pris par Judith en flagrant délit d'adultère, Abel affirme: "Nous ne faisions que le mal, et Dieu vit que cela était bon" (p. 59), employant ainsi une phrase de la Genèse dans un nouveau contexte sémantique.

Ce dernier cas d'intertextualité biblique n'est pas isolé; les liens qui unissent Don Quichotte aux livres saints sont multiples: nombreuses citations, concordances diégétiques (comme la scène du Déluge) et, surtout, concordances actorielles. Nonobstant les personnages qu'un bref relevé onomastique met en évidence (Abraham, Thadée, Moïse, Borromée), le réseau intertextuel se construit autour de trois figures bibliques: Abel, Caïn-Steven et Judith. Dans la Bible, Judith tue un général assyrien du nom d'Holopherne, après avoir feint de se laisser séduire par lui, et libère ainsi la ville de Béthulie. Dans Don Quicbotte, Judith abandonne Steven pour Abel, mais donne l'impression de n'avoir jamais cessé d'aimer le premier. Si ce triangle amoureux occupe une place importante dans l'intrigue, il n'explique cependant pas la jalousie qui déchire les deux frères ennemis. La relation ambiguë entre Abel et Steven remonte à l'enfance, renforçant ainsi le caractère primaire du récit familial.

Sur un plan pragmatique, la cause de dissension est le statut d'écrivain. Par rapport à la Bible, il y a interversion actantielle puisque c'est Abel qui jalouse son frère. Cette rivalité, paradoxalement, se nourrit du détachement de Steven. Alors qu'Abel exerce son métier de romancier avec l'ambition avouée d'être celui qui écrira le Grand CEuvre, Steven ne songe aucunement à lui dérober le titre d'écrivain familial ou national, ne vivant que pour la pureté de la poésie. Dans la Bible, la jalousie a pour origine l'acceptation par Dieu des offrandes d'Abel, qui est berger, et son refus de celles de Caïn, qui cultive la terre. En extrapolant quelque peu, on pourrait donc voir dans l'agriculteur laborieux la figure du romancier et dans le berger rassembleur, celle du poète national.

Cette opposition entre le roman et la poésie, comme plusieurs critiques l'ont noté, traverse tout entière l'œuvre de Victor-Lévy Beaulieu. Pierre Nepveu a bien montré comment chez VLB, et dans une grande part de la production québécoise contemporaine, 
la conception romantique de l'écriture est indissociable d'un sentiment d'inaccomplissement du roman par rapport à la poésie. $\mathrm{Si}$, dans Don Quicbotte de la démanche, Abel exprime trop ouvertement sa nostalgie de la poésie pour qu'il soit nécessaire d'en citer ici des exemples, mentionnons que cette valorisation est partagée par Judith, qui déclare à Abel: «Au moins, Nelligan était un poète, lui!n (p. 73), et par VLB lui-même, qui, à la fin de Melville, décrit son œuvre à venir, La grande tribu, comme "le lieu de la poésie épique et lyrique, seule capable de faire apparaître l'histoire " ${ }^{10}$. Cet inachèvement romanesque peut se lire comme un miroir de l'inachèvement québécois, tant sur le plan historique que littéraire, et devient en fait la matière même du roman ${ }^{11}$

Cette fascination pour l'épique culmine dans une autre figure intertextuelle, celle de James Joyce. Je ne reviendrai pas sur les liens féconds qui unissent VLB, à la fois comme romancier et comme critique, et l'écrivain irlandais. Mais dans le cadre de cette analyse, il faut s'arrêter à la fonction de cette figure dans le roman. Dans une récente entrevue, VLB déclarait: "Pour moi, Joyce incarne l'absolu du poète: il a exploré toutes les formes du langage jusqu'à créer un métalangage, une métapoésie " 12 .

Le texte de Don Quicbotte de la démanche désigne Steven comme le double de Joyce. Le frère d'Abel est l'auteur d'une traduction de Finnegans Wake, habitait à Paris face à la Bibliothèque Sainte-Geneviève où, nous dit-on, l'écrivain irlandais allait souvent et cite volontiers les œuvres de ce dernier. Abel demeure cependant celui qui attribue en rêve ces citations à Steven: il est donc celui qui réalise l'identification. C'est d'ailleurs dans l'un de ces rêves que Steven se fait attaquer par un cochon qui avale son exemplaire d'Ulysse. Plusieurs chapitres plus loin, on apprend que, dans son enfance, Abel avait été condamné à nettoyer les cochons tout un hiver pour s'être moqué de Steven, qui avait pleuré la mort de la Grand-mère. Abel prend donc symboliquement sa

10 Cité par Pierre Nepveu dans "Abel, Steven et la souveraine poésie", Études françaises, vol. XIX, n० ${ }^{\circ}$, printemps 1983, p. 32.

11 Comme l'écrit Nepveu: "Les gens de Parti pris avaient décrété l'CEuvre impossible, mensongère. Beaulieu montre que l'impossibilité de l'œuvre peut se vivre dans le roman comme désir passionné de l'Euvre, comme affirmation redoublée de sa possibilité à venir *, op.cit., p. 38.

12 Catherine Lamy et Jean Morency, "Tolstoï, Joyce, Melville et les autres... Entretien avec Victor-Lévy Beaulieu ", Nuit blancbe, $\mathrm{n}^{\circ}$ 51, mars-avril-mai 1993 , p. 50. 
40

revanche sur la sensibilité poétique et joycienne: "Je te détestais, Steven, car je voyais bien que tu es meilleur que moi et grand comme je ne deviendrai jamais, toi authentiquement poète, croyant encore à des choses que je récuse" (p. 115).

Si Abel voit en Steven l'absolu de la poésie, il n'a pas luimême renoncé à cet idéal (après tout Joyce était romancier). Cela explique pourquoi il cite à maintes reprises la célèbre périphrase joycienne de l'écrivain: "Tisse, tisseur de vent", et c'est pour cela qu'il force certaines comparaisons. Ce réseau intertextuel comprend également Judith, qui est identifiée à la fois à Molly Bloom, la femme aimée et adultère d'Vlysse, et à Anna Fluvia Plurabelle de Finnegans Wake. Enfin, la nuit au cours de laquelle Abel croit mourir renvoie à la veillée funèbre qui donne son titre à Finnegans Wake.

Cette rivalité qui oppose Abel à Steven ainsi que le roman à la poésie se double d'une autre rivalité fratricide. Dans le cauchemar familial dont nous avons précédemment parlé, on observe un sentiment de culpabilité chez Abel, qui se reproche, par l'entremise de Jos, la nature égocentrique de sa démarche littéraire: "Je te volerai ton monde, Abel, car tu es indigne de lui, ne pensant qu'à t'en servir alors qu'il aurait fallu que tu le serves. Le projet du grand Euvre, c'est moi qui l'accomplirai" (p. 227). Abel, est donc hanté par la relation problématique de l'écrivain québécois avec le Texte national ${ }^{13}$.

Mais la fonction même de la littérature est aussi remise en question, Jos rejetant l'écriture au profit des traditions (il joue de l'harmonica) èt de l'action révolutionnaire (il est à la tête d'un mouvement révolutionnaire, La Secte des Porteurs d'eau, qui n'est pas sans rappeler le FLQ):

Par mon chant, dit Jos lorsqu'il s'arrêta de jouer, je puis recréer notre monde et l'assurer dans sa pérennité. [...] Ce que je veux,

13 Rappelons-nous le mot d'ordre de Jacques Godbout: "Un auteur québécois n'a donc de valeur que s'il participe de façon intense au TEXTE UNIQUE, mais aussi il ne pourra exister que s'il découvre les clefs du TEXTE NATIONAL. La littérature québécoise, en somme, est une chanson à répondre qu'on apprend au berceau, un air de folklore que l'on turlute toute sa vie. Et les écrivains québécois qui ne savent pas turluter (parce qu'ils ont trop appris de chansons françaises) continuent d'écrire mais en vain: ils sont de nulle part." "Écrire", Liberté, vol. XIII, n ${ }^{\circ} 4$, novembre 1971, repris dans Le réformiste, Quinze, 1975, p. 151. 
c'est que le rêve de nous-mêmes [...] s'accomplisse, non dans quelques mots alambiqués, destinés à moisir sur une étagère, mais dans un quotidien renouvelé. Pour ce faire, nous avons besoin de toutes les énergies et de tout ce passé qui vit en nous. (p. 229)

Si le débat entre l'art et l'action informe la littérature québécoise depuis Prochain épisode d'Aquin et Le couteau sur la table de Godbout, il prend un tour nouveau avec l'invocation du passé (qu'on peut lire comme une métaphore du récit de fondation), qui n'est pas sans dimension messianique. Faisant rapport sur la secte, Abraham Sturgeon affirme: "Le credo est clair: le Québec constitue le dépotoir de l'humanité, un formidable bouillon de culture, la matrice d'une nouvelle civilisation." (p. 155) Quant à Jos, il affirme n'avoir "encore rien dit non plus des centres de recherches, de la Bibliothèque totale qu'il nous faudra constituer, de tous ces morts à déterrer et à interroger. Et rien dit aussi du nouveau cosmos dont le Québec créera la configuration définitive" (p. 231). Ces propos rappellent l'historien britannique Arnold Toynbee, selon qui deux peuples sur la planète pouvaient relever le défi de la nouvelle civilisation et apporter une réponse à l'humanité: les Chinois et les Canadiens français ${ }^{14}$. Cette affirmation étonnante eût un impact considérable au Québec et il ne serait pas surprenant que VLB s'en soit inspiré. Mais ce qui présente un intérêt particulier, outre que Toynbee ait été l'un des premiers à utiliser le terme postmoderne ${ }^{15}$, c'est l'amalgame de la littérature de la Bibliothèque (trait postmoderne), du discours du progrès historique (trait moderniste) et du passé familial (trait fondateur).

Un écrivain québécois qui a su réconcilier traditions populaires, passé, projet politique et pratiques narratives contemporaines est sans doute Jacques Ferron, auquel Don Quichotte de la démanche rend hommage par une série de mécanismes intertextuels. En le faisant apparaître dans le ciel de Morial, VLB consacre Ferron et un de ses romans. De plus, en incluant

14 Cité par Jacques Godbout dans "Pour une révolution culturelle", Le réformiste, Quinze, 1975, p. 132.

15 Sur cette question, on peut consulter Janet Paterson, Moments postmodernes dans le roman québécois, Presses de l'Université d'Ottawa, 1990, p. 10-11, ainsi que mon ouvrage, Les mots des autres. La poétique intertextuelle des ceuvres romanesques de Hubert Aquin, Sainte-Foy, Presses de l'Université Laval, coll. "Vie des lettres québécoises", 1992, p. 247. 
42

Archibald Scott dans la procession, il renvoie à un second roman, La nuit, et reproduit un mécanisme ferronien, le travestissement onomastique $^{16}$. Don Quichotte de la dêmanche et L'amélanchier ont également plusieurs points en commun: un récit familial qui tient lieu de genèse historique, un bungalow de banlieue qui départage le réel de l'imaginaire, un père qui travaille dans un institut psychiatrique, etc. D'autres travaux définiront sans doute mieux ce réseau intertextuel que je ne le fais ici, mais un de ses effets importants est de légitimer le discours littéraire québécois, de l'inscrire dans la littérature universelle.

Dans un essai de classification du roman québécois, Laurent Mailhot distingue le "roman de l'écriture" et le "roman de la parole "17. L'œuvre de Ferron, en ce qu'elle est à la fois hautement intertextuelle et traversée par une authenticité qui plonge dans le passé, semble opérer la synthèse des deux types. Dans son désir de totalité, l'œuvre de Victor-Lévy Beaulieu cherche à dépasser ce fragile équilibre, mais est condamnée par l'Histoire à ce paradoxe: un récit de fondation qui s'ouvre à jamais au récit de l'autre, qui perpétue la quête identitaire. Dans cette contradiction réside peut-être la spécificité du postmodernisme québécois. Comme le dit Abel: "Écrire, ce n'était que rouvrir une blessure" (p. 22).

16 Dans La nuit, Frank Scott, le critique et traducteur de poètes québécois, devient Frank Archibald, qui devient, dans le roman de VLB, Archibald Scott. Ce titre renvoie également aux rites nocturnes d'Abel.

17 Laurent Mailhot, "Romans de la parole (et du mythe)", Canadian Literature, $\mathrm{n}^{\circ} 88,1984$, p. 84-90. 